\title{
Assessment of Periodontal Health in Nepalese Orthodontic Patients
}

\author{
Dr Bhageshwar Dhami,,' Dr Priti Shrestha,, Dr Rabindra M Shrestha,3 Dr Jyoti Dhakal4 \\ Lecturer, Dept of Periodontics, ${ }^{3}$ Asso Prof, ${ }^{4}$ Lecturer, Dept of Orthodontics \\ Kantipur Dental College, Kathmandu, Nepal \\ Lecturer, Dept of Periodontics, KIST Medical College, Lalitpur, Nepal
}

\section{ABSTRACT}

Objective: To assess periodontal status of the patients undergoing orthodontic treatment and to compare periodontal health of patients with and without orthodontic treatment.

Materials \& Method: A cross sectional study was done on 100 patients (50 orthodontic and 50 non-orthodontic). CPITN (Community Periodontal Index for Treatment Need) was used to assess the periodontal health of indexed teeth. SPSS version 17 and Chi Square test were used to analyze and compare the data.

Result: There was a statistically significant association in CPITN score between orthodontic and non-orthodontic patients $(p<0.01)$.

Conclusion: Patients undergoing orthodontic treatment have increased plaque accumulation and probing depth that may be associated with periodontal destruction. Patient motivation to maintain oral hygiene and regular scaling will minimize hazardous effects in orthodontic patients.

Key words: CPITN, fixed orthodontic treatment, gingivitis, pocket depth

\section{INTRODUCTION}

Gingivitis is an inflammation of gums surrounding the teeth. Gingivitis is one of the periodontal diseases that affects health of the periodontium.' Fixed orthodontic appliances may impair plaque removal, proper oral hygiene, and affect gingival health. ${ }^{2}$ Gingivitis may develop in patients who do not institute proper oral hygiene measures and it can become quite profound in 21 days. ${ }^{3}$ Patients often exhibit gingival hypertrophy, bleeding, increased plaque accumulation, and calculus formation during orthodontic treatment. ${ }^{4}$ Thus, oral hygiene measures are recommended because bands, brackets, ligature wires, and elastics encourage the accumulation of microbial flora and food residues. ${ }^{5.6}$ In time, the plaque accumulation around orthodontic appliances may cause periodontal disease and caries. ${ }^{\text {? }}$

The aim of this study was to assess periodontal status of the patients undergoing orthodontic treatment and to compare periodontal health of patients with and without orthodontic treatment.

\section{MATERIALS AND METHOD}

A cross sectional study was performed on 50 orthodontic patients i.e. ortho group and 50 nonorthodontic patients i.e. non-ortho group selected from Orthodontic and Periodontic Departments of Kantipur Dental College, Kathmandu, Nepal. Patients included in the study were in age range of 15-28 years of both sexes with more than 3 months duration after starting the orthodontic treatment. Patients with any systemic disease, generalized periodontal problems/ disease, cyst, cleft or congenital malformations, and patients receiving removable appliance treatment were excluded from the study.

All patients were assessed by using CPITN (Community Periodontal Index for Treatment Need $)^{8}$ on the index teeth $(16,11,26,36,31,46)$. The recording of data was based on the WHO Oral Health Assessment Survey Form (proforma). A questionnaire was used to assess the use of oral hygiene aids by the participants. 


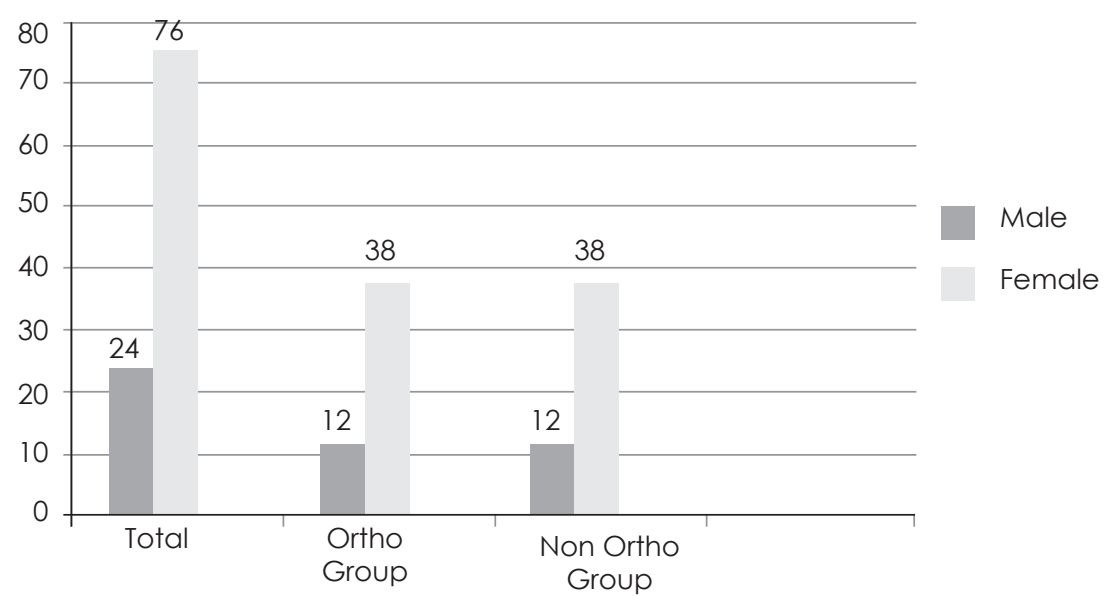

Figure 1: Gender distribution among the subjects

\section{Examination Procedure}

Each subject was examined on dental chair under adequate natural light using mouth mirror and WHO CPITN Probe. The Examination was performed in a systemic manner beginning from maxillary right sextant. The tip of the probe was gently inserted parallel to the long axis of the tooth, between the tooth and gingiva starting at the distobuccal surface of index tooth, to the full depth of the sulcus or pocket and the probing depth was read by observing the black position of the black band. The sites probed were the distal, midline and mesial on both facial and lingual/palatal surfaces. The appropriate highest score for each sextant was determined and recorded in the appropriate box.

The following CPI coding system was used to record periodontal status: 0 , healthy periodontium; 1 , bleeding observed after probing; 2, calculus detected during probing, but the black band on the probe was visible; 3 , pocket $4-5 \mathrm{~mm}$ (the black band on the probe is within the gingival margin); 4 , pocket $\geq 6 \mathrm{~mm}$ (black band on the probe not visible); $X$, excluded sextant (>2 teeth present); and 9 , not recorded.

All recorded data were statistically analyzed using SPSS version 17 and various comparisons were performed by chi square test.

\section{RESULTS}

Out of 100 patients examined, 24 were male and 76 were female; detail distribution of subjects is given in Figure 1. Age range of the patients was 15 to 28 years. Out of 50 patients in ortho group; 30 patients i.e. $60 \%$ had periodontal pocket (CPITN score 3 \& 4). Table 1 shows distribution of CPITN Score between orthodontic and non-orthodontic patients. While comparing the CPITN score between ortho group and non-ortho group, $81.1 \%$ had periodontal pocket (CPITN score 3 \& 4 ) in ortho group where as only $18.9 \%$ had periodontal pocket (CPITN score 3 \& 4) in non-ortho group. There is statistically significant association between advancement of periodontal disease in ortho and non-ortho groups $(p<0.01$ ) (Table 2$)$.

Table 1: Distribution of CPITN Score among orthodontic and non-orthodontic patients

\begin{tabular}{|l|c|c|c|c|c|c|}
\hline \multicolumn{1}{|c|}{ CPITN } & Score 0 & Score 1 & Score 2 & Score 3 & Score 4 & Total \\
\hline Orthodontic patient & 2 & 14 & 4 & 29 & 1 & 50 \\
\hline Non-orthodontic patient & 11 & 24 & 8 & 7 & 0 & 50 \\
\hline
\end{tabular}

Table 2: Comparison of CPITN Score between orthodontic and non-orthodontic patients

\begin{tabular}{|l|c|c|c|}
\hline \multicolumn{1}{|c|}{ CPITN } & Score $\mathbf{0 , 1 , 2}$ & Score 3,4 & p-value \\
\hline Orthodontic patient $(\mathbf{N}=\mathbf{5 0})$ & $20(31.7 \%)$ & $30(81.1 \%)$ & $0.000^{*}$ \\
\hline Non-orthodontic patient $(\mathbf{N}=\mathbf{5 0})$ & $43(68.3 \%)$ & $7(18.9 \%)$ & \\
\hline
\end{tabular}

* Statistically significant 
Comparison of patients using and not using interdental aids in all subjects is shown in Figure 2. The study revealed that, only $3 \%$ patients using interdental aids had periodontal pocket (CPITN score 3 \& 4); whereas $34 \%$ had periodontal pocket who were not using interdental aids (CPITN score 3 \& 4). There is significant association in advancement of periodontal disease between interdental aids users and non-users among all patients ( $<<0.01)$ (Table 3).

Among orthodontic patients only 3(6\%) using interdental brush had periodontal pocket (CPITN score 3 \& 4), whereas $27(54 \%)$ patient had periodontal pocket (CPITN score 3 \& 4) who were not using it (Figure 3).

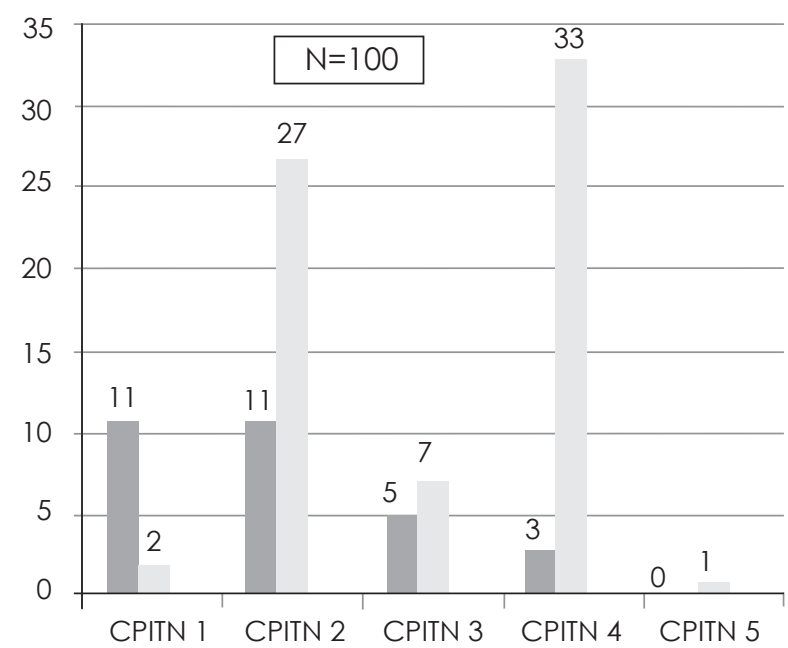

Using interdental brush Not using interdental brush

Figure 2: Distribution of CPI score and use of interdental aids

Table 3: Comparison of CPITN Score between interdental aids uses and non users

\begin{tabular}{|l|c|l|c|}
\hline \multicolumn{1}{|c|}{ CPITN score } & Score $0,1,2$ & Score 3,4 & p value \\
\hline Using interdental aids ( $N=30)$ & $27(90 \%$ & $3(10 \%)$ & \multirow{2}{*}{$0.000^{*}$} \\
\cline { 1 - 3 } Not using interdental aids $(\mathrm{N}=70)$ & $36(51.4 \%)$ & $34(48.6 \%)$ & \\
\hline
\end{tabular}

*Statistically significant

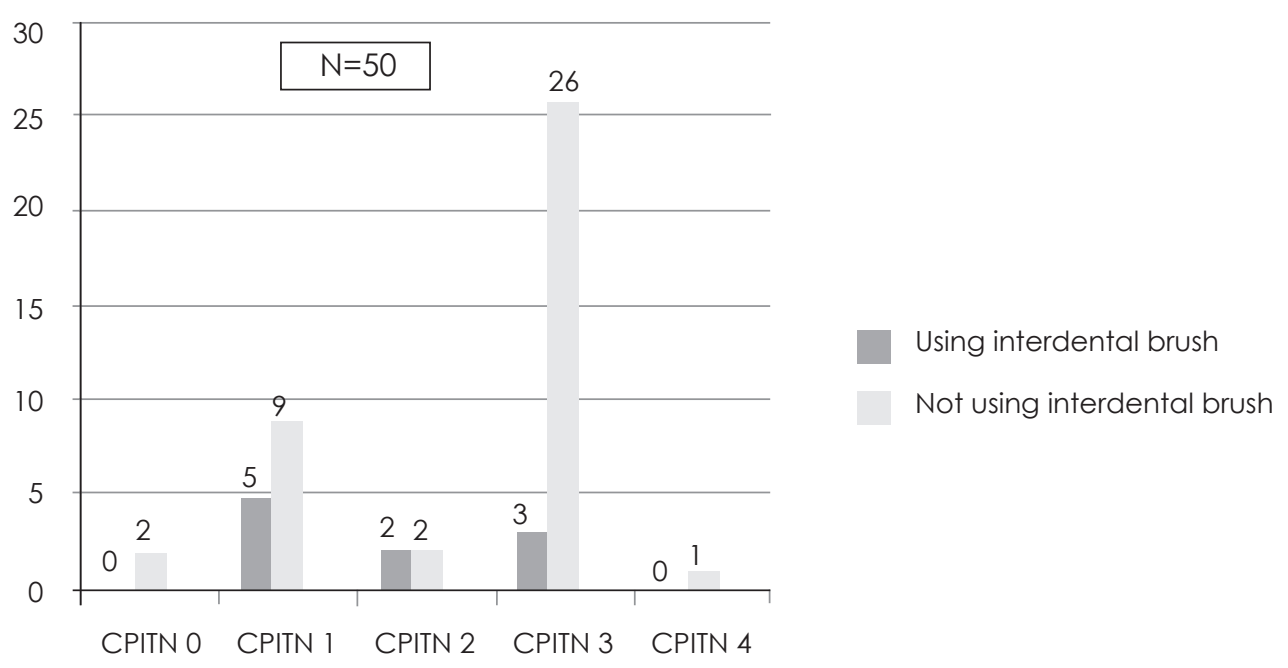

Figure 3: CPI scores among orthodontic patients using interdental aids 


\section{DISCUSSION}

This study examined the periodontal status of orthodontic patients and non-orthodontic patients. The data of patients receiving fixed orthodontic treatment showed an increase in CPITN score. It agrees with the results of Naranjo et al who reported that the placement of brackets influenced the ecological environment of retentive sites by the accumulation of biofilm. There was a marked increase in Plaque and Gingival Index in the experimental group resulting in more bleeding and inflammation that deteriorated periodontal condition. 9 Similar results were observed by Ristic et al, as there was a marked increase in both clinical and microbiological parameters in 3 months time after the fixed appliance was placed. ${ }^{10}$

There was a significant difference in the CPITN scores between ortho and non-ortho groups. Patients using interdental aids had significantly lower CPITN score while comparing with patient not using interdental aids. CPITN score was more in orthodontic patient who do not use interdental aids. The accumulation of dental plaque on orthodontic appliances cause difficulty for patients to maintain oral hygiene. Thus it may be believed that the placement of fixed orthodontic appliances can result in accumulation of plaque, increase in pocket depth leading to the increase of CPITN score.

Baer et $\mathrm{a}^{11}$ suggested that interdental areas are especially more periodontally affected in orthodontic patients. Itreflects and assures ability of fixed orthodontic appliance accumulates dental plaque which is the initiating factor for periodontal disease. These results agree with the results from other studies. ${ }^{12}$ In clinically healthy human gingiva, a sulcus of some depth can be found. The depth of periodontal sulcus was determined differently, some studies reported the depth of $1.5 \mathrm{~mm}$ and others reported $1.8 \mathrm{~mm} \cdot{ }^{13,14}$ Radiographs indicate areas of bone loss where pockets may be suspected. ${ }^{15}$ Zachrisson et $a^{16}$ reported an increase in probing depth and a slight loss of attachment around teeth of patients who underwent orthodontic treatment with fixed orthodontic appliances.
Plaque is the primary etiological agent in almost all periodontal and gingival conditions. Orthodontic appliances present a challenge to the proper removal of plaque from the tooth and gingival surfaces. Organisms commonly present in an early plaque are Gram-positive rods and cocci. Over time, these organisms are replaced by more Gram-negative and anaerobic organisms, which may initiate a periodontal reaction. ${ }^{17}$ Orthodontic appliances cause mechanical plaque traps where plaques may evolve into a pathological state, because adequate oral hygiene measures are more difficult to achieve during orthodontic treatment. Thus, plaque control must be emphasized as the most important factor in preserving periodontal health in patients undergoing orthodontic therapy. Motivating and making them to practice oral hygiene measures in young age groups will certainly enhance the levels of oral hygiene standards. ${ }^{8,19}$ Powered and inter-dental tooth brushes and special types of floss have been shown to improve plaque control in orthodontic patients. ${ }^{20}$

\section{CONCLUSION}

During the orthodontic treatment, plaque score and pocket depth increases which results in periodontal destruction but it did not cause any severe bone loss, tooth mobility and tooth loss. The gingiva was in a relatively stable situation, and no remarkable recessions were reported. During orthodontic treatments the patient's oral hygiene should be maintained in high levels so that negative effects can be minimized. Patient motivation and oral hygiene education are essential elements for successful orthodontic outcome. Therefore, appropriate oral hygiene methods and interdental aids should be used to control plaque.

\section{ACKNOWLEDGEMENT}

We would like to thank Dr Sujita Shrestha, Dept of Community \& Public Health Dentistry, $\mathrm{KDCH}$ for her assistance in statistical analysis, to the patients, all the staffs and interns of departments of Periodontics and Orthodontics.

\section{OJN}




\section{REFERENCES}

1. Stuteville $\mathrm{OH}$. Injuries caused by orthodontic appliances and methods of preventing these injuries. JADA 1937;24:1494-507

2. Anhoury P, Nathanson D, Hughes CV, Socransky S, Feres M, Chou LL. Microbial profile on metallic and ceramic bracket materials. Angle Orthod 2002;72:338-43

3. Atack NE, Sandy JR, Addy M. Periodontal and microbiologicalchanges associated with the placement of orthodontic appliances. A review. J Periodontol 1996;67:78-85

4. Turkkahraman H, Sayin MO, Bozkurt FY, Yetkin Z, Kaya S, Onal S. Archwire ligation techniques, microbial colonization, and periodontal status in orthodontically treated patients. Angle Orthod 2005;75:231-6.

5. Kilicoğlu H, Yildirim M, Polater H. Comparison of the effectiveness of two types of toothbrushes on the oral hygiene of patients undergoing orthodontic treatment with fixed appliances. Am J Orthod Dentofacial Orthop 1997;1 11:591-4.

6. Axelsson P, Lindhe J, Nystrom B. On the prevention of caries and periodontal disease. Results of a 15-year longitudinal study in adults. J Clin Periodontol 1991;18:182-9.

7. Lo BA, Di Marco R, Milazzo I, et al. Microbiological and clinical periodontal effects of fixed orthodontic appliances in pediatric patients. New Microbiol 2008;31:299-302.

8. Ainamo J, Barmes D, Beagrie G, Cutress T, Martin J, Sardolnfirri J. Development of the World Health Organization (WHO) Community Periodontal Index of Treatment Needs (CPITN). Int Dent J 1982:32:281 -291.

9. Naranjo AA, Trivino ML, Jaramillo A, Betancourth M, Botero JE. Changes in the subgingival microbiota and periodontal parameters before and 3 months after bracket placement. Am J Orthod Dentofacial Orthop 2006;130:275 e17-22.

10. Ristic M, Vlahovic Svabic M, Sasic M, Zelic O. Clinical and microbiological effects of fixed orthodontic appliances on periodontal tissues in adolescents. Orthod Craniofac Res 2007;10:187-95.

11. Baer PN, Coccaro PJ. Gingival enlargement coincident with orthodontic therapy. J Periodont 1964;35:436-9.

12. Nunn ME. Understanding the etiology of periodontitis: an overview of periodontal risk factors. Periodontol-2000 2003;32:1 1-23.

13. Anerud A. The effect of preventive measures upon oral hygiene and periodontal health. Thesis. University of Oslo 1970.

14. Frandsen A, Barbano JP, Suomo JD, Chang JJ, Burke AD. The effectiveness of the Charter's scrub and roll methods of tooth brushing by professionals in removing plaque. Scand J Dent Res 1970;78:459-63.

15. Diamanti-kipioti A, Gusberti FA, Lang NP. Clinical and microbiological effects of fixed orthodontic appliances. J Clin Periodontol $1987 ; 14: 326-33$

16. Zachrisson S, Zachrisson BU. Gingival condition associated with orthodontic treatment. Angle Orthod 1972;42(1):26-34.

17. Eckley B, Thomas J, Crout C, Ngan P. Periodontal and microbiological status of patients undergoing orthodontic therapy. Hong Kong Dent J 2012;9:11-20.

18. Polson AM, Subtelny JD, Meitner SW, Polson AP, Sommers EW, Iker HP et al. Long term periodontal status after orthodontic treatment. Am J Orthod Dentofac Orthop 1988; 93:51-58.

19. Eliasson LA, Hugoson A, Kurol J, Siwe H. The effects of orthodontic treatment on periodontal tissues in patients with reduced periodontal support. Eur J Orthod 1982; 4:1-9

20. Diedrich P, Rudzki-Janson I, Wehrbein H, Fritz U. Effects of orthodontic bands on marginal periodontal tissues. A histological study on two human species. J Orofac Orthop 2001;62:146-56. 